\title{
Online instrument systems in reality for remote wiring and measurement of electronic in e-learning from labVIEW+NI ELVIS II vs embedded system+web services
}

\author{
Fahd Ouatik ${ }^{1}$, Mustapha Raoufi ${ }^{2}$, Farouk Ouatik ${ }^{3}$, Mohammed Skouri ${ }^{4}$ \\ ${ }_{1,2,3,4}$ Laboratory of Physics High Energy and Astrophysics, Marrackech Faculty Science Semlalia, \\ Cadi Ayyad University, Morocco \\ ${ }^{3}$ Laboratory Mathematics and Informatics, Sultan Moulay Slimane University, Beni Mellal, Morocco
}

\begin{tabular}{l}
\hline \hline Article Info \\
\hline Article history: \\
Received Oct 14, 2019 \\
Revised Sep 5, 2020 \\
Accepted Oct 15, 2020 \\
\hline
\end{tabular}

\section{Keywords:}

E-learning

Engineering education

Labview

NI ELVIS II

Remote laboratory

Web tools

\begin{abstract}
Recently The prestigious universities of the world strive and aim to computerize their distance education service and more specifically the remote practical work, which allows students to manipulate electronic experiments via the web, for to solve a set of problems: pedagogical, management, security, restriction by time and place and the problems the overcrowding of students in universities. This paper will describe the E@SLab system developed by the university Cadi Ayyad member of the e-live project funded by EU in the frame of ERASMUS+E@SLab is based on the latest technologies of development and respects educational and pedagogical standards. E@SLab offers 2 versions different of remote laboratory: first version (s1) is an embedded system its part software is node js+Ubuntu and the part hardware a card pcduino or raspebery. The second version (s2) is based on LABview and its hardware part is the NI ELVIS II pedagogical map. In this paper, we will compare and discuss the architecture, performance of the 2 versions of E@SLAB and review other famous approaches NetLab, VISIR, for comparing with E@SLab with the intention show its singularity.
\end{abstract}

This is an open access article under the CC BY-SA license.

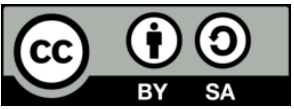

\section{Corresponding Author:}

Fahd Ouatik

Laboratory of Physics, High Energy and Astrophysics

Marrackech Faculty Science Semlalia

Cadi Ayyad University, Morocco

Email: fahd.ouatike@gmail.com

\section{INTRODUCTION}

Remote laboratory comes for to supplement and enrich the offer of scientific training and practical and experimental teaching in the fields of engineering science for resolute a sets of constraint in the universities such as overcrowding of students in front of the limited resources of the universities. Remote laboratory $[1,2]$ also give a new dimension, even a new direction, to the role of the teachers. Concept of a remote laboratory makes it possible to overcome not only the constraint of place, but also the often rigid schedules and Appointment associated with the practical work for students [3, 4]. This new possibility of performing remote laboratory for practical works present a flexible approach of learning and also extends the didactic role means of university [5].

Development of remote laboratory installations is also motivated by the need to offer practical education to a big number of students, in spite of limited equipments and human resource. Being able to access remote laboratory resources ultimately allows sharing these installations with other universities or 
training institutions, contributing [6, 7]. Development and installation E@SLab for remote laboratory has been successfully used in the Faculty Semlalia of Sciences of Cadi Ayyad University and these partnerships for more than two years in electronics courses to illustrate important concepts through real demonstrations [8]. The practical work we have developed concerns the analog electronics including the operational amplifier, RLC circuit, Multivibrators [9]. These Practical works we have developed in two ways and with 2 different version of E@SLab system (S1 and S2 version), each version has features, advantages and disadvantages. The first solution S1: is an embedded system its software part is the node js technology and OS is ubuntu and the hardware part is a PCduino or Raspebery card [10]. The second solution S2 is based on LABview the NI ELVISS II card.

In this paper we are going to describe the web and communication technologies used in the development of S1 and S2 system as well their architecture and their compatibility with the pedagogic and didactic approach [11]. And we discuss the reasons for choosing each technology in each system and discuss the advantages and disadvantages of each system. This paper review other famous systems NetLab, and VISIR, face E@SLab in aims highlight its singularity $[12,13]$.

\section{ONLINE LABORATORY S1 VERSION OF E@SLAB USING EMBEDDED SYSTEM+TOOLS OF WEB SERVICES}

\subsection{Architecture of E@slab v1}

Architecture of E@Slab distance laboratory is distributed in tow servers presented in Figure 1, in server1 hosted the information system of university and the management platform $[14,15]$, via this platform students find online course, take appointment for a practical work. In server 2 hosted the user interface of remote and wiring is an application developed with Node js that allow students to handle PW, server 2 is a PCDUINO Card, with this card we can send and receipt electrical signal to control many other types of equipment to be correctly connected and several measuring devices and instruments $[16,17]$.

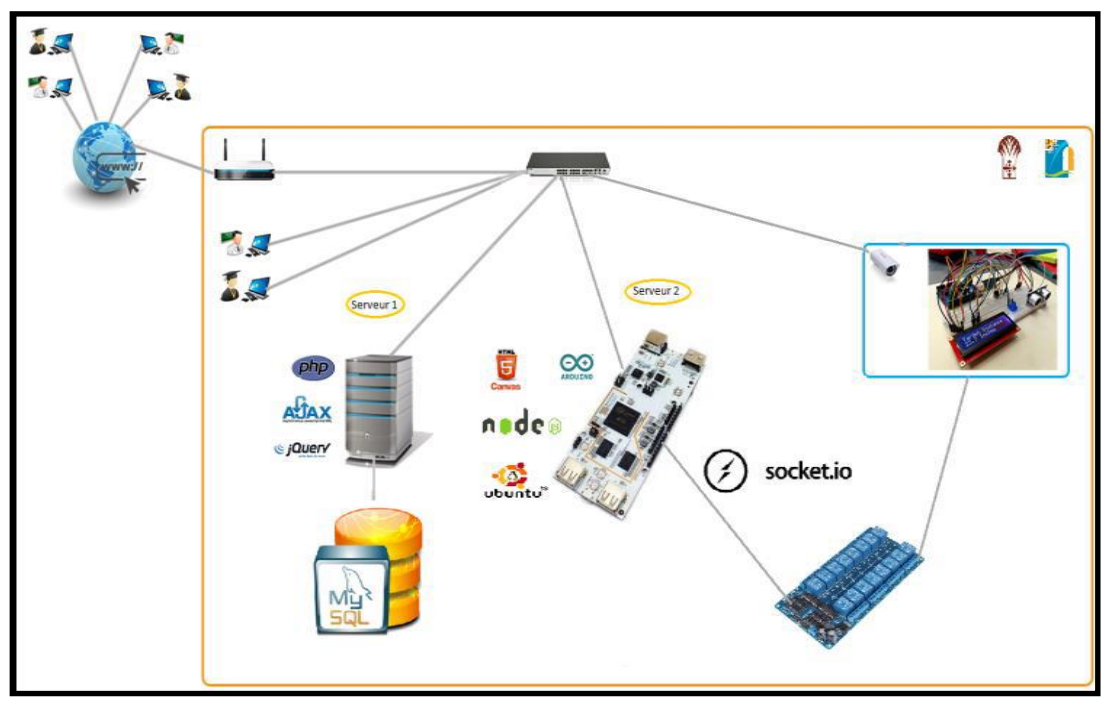

Figure 1. E@SLAB architecture

Process of using E@Slab:

- $\quad$ First teacher connect to the platform and authenticate, put in the platform the practical work that the students must do (theoretical and practical question), put a set of slots to be reserved by students.

- Students connect to their account in the platform and check their box of notification, they find a set of niches for choose the suitable one.

- If it's the time a user interface appears to student allow to do the practical work and control the real measure device and equipment [18].

\subsection{User interface for remote and wiring}

Students will assemble the electrical diagram by wiring between devices of the circuit, and read value of tension from measurement device, all via the web Figure 2 The most important technologies used in 
development the user interface for remote and wiring is html5, Canvas, Node.js, socket [19, 20] and the hardware part is an embedded system. To have animation on the user interface for student can drag and drop devices and wiring them, we choice to use Canvas of html5 for to have compatibility with all web browser.

For programming with Canvas that need to use many bibliotics of JavaScript, so to not weigh down our server2 we used the Node.js framework, allow installing a web server on our embedded system [21]. Node.js is allows to execute JavaScript inside the server and not at the customer, is used the motor of google chrome V8 that makes execution very faster. This framework is already used to implement remote laboratory system [22, 23], but in our approach the websocket library that allows the transfer bidirectional of information between the customer and the server, this protocol its used in the latest work, but it not exploited the interest of this protocol; its only used for sending the request from the customer to the server, but that we can to do just with Ajax language. But with Ajax [24] it is always the customer who request and the server resend. The server cannot decide by itself to send information to the customer.

With webSocket that become possible, and we will use this important thing for sending request from server itself our pcduino card for to protect electronic device of the practical work; for example if the level voltage apply electronic components to the input that do not support high voltage the server must send alert to student to take attention for protect electronic devices [25].

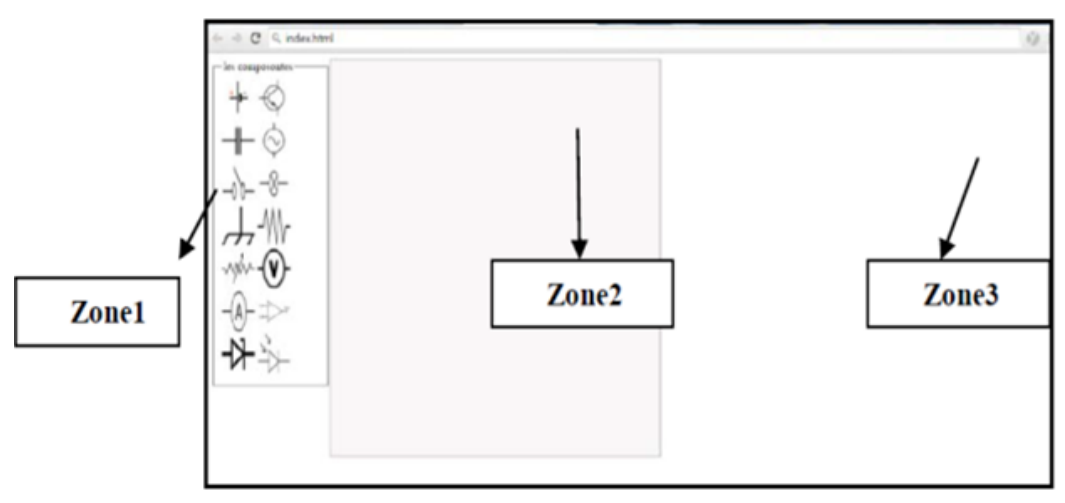

Figure 2. User interface for remote and wiring

Zone 1: Present all electronic components that the student drag and drop in the workbench for wiring between them.

Zone 2: Is the workbench where students must wiring between devices and adjust voltage sources and measuring devices.

Zone 3: In this zone will display the interface of measuring tools or power for adjust voltage sources and measuring devices.

\section{ONLINE LABORATORY S2 VERSION OF E@SLAB USING NI LABVIEW AND NI ELVIS II}

This second architecture Figure 3 present the role of National Instrument of software and hardware in the building an modern learning technologies such as the educational card platform NI ELVIS II [26] and NI LabView [27]. LabVIEW is a development environment specializing in industrial computing and embedded systems. Its particularity is that it relies on the language G, created by National Instruments, which is entirely graphic. It makes it possible to create complex software while facilitating the programming and thus reducing the development time [28]. Thanks to its libraries of functions dedicated to data acquisition, instrumentation, mathematical analysis of measurements, but also thanks to the rapid creation of quality graphical interfaces and simplified coding, the engineer has more than time to focus on the business functions of instrumentation and measurement processing. LabVIEW is particularly recommended for developing control systems, supervision and test and measurement benches.

LabVIEW allows you to program on many different targets thanks to its many libraries, you can interface and control the following cards and devices: VXI, PXI, Compact PCI, PCI, Express PCI, Express PXI, USB, FireWire, GPIB, CAN RS 232, 422, 485 TCP/IP, Bluetooth, WIFI, Programmable Controllers (PLC), Modbus, Profibus [29]. This version S2 of E@Slab laboratory is based on the Elvis II card. The NI ELVIS II Figure 4 is an environment educational laboratory of electronic card developed specifically for using by student in a context educational in electronic engineering. on this card, teachers can help students to 
do the practical work and experience of electronic for student get all technic skills. NI ELVIS II is a modular integrated with 12 of the most commonly used instruments in the laboratory, including an SCOPE, digital multimeter, Power and function supply, variable power generator, and Bode analyzer Figure 5. This card is connected directly to PC who is installed Labview for controlling all integrated module of NI elvis. NI ELVISmx is the software integrated in labview that supports, control and command Elviss card and the NI myDAQ card [30].

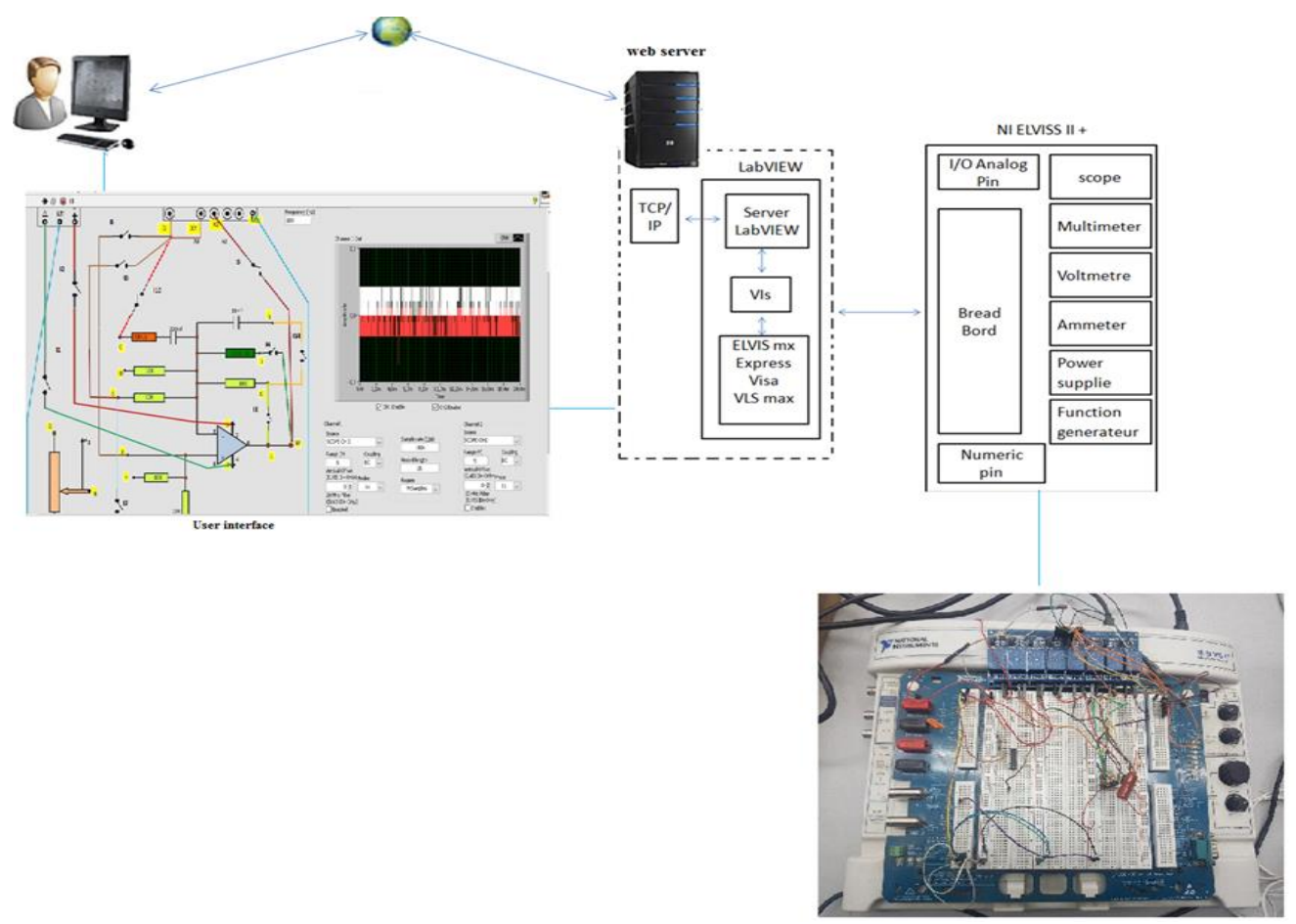

Figure 3. Architecture and technologies system S2

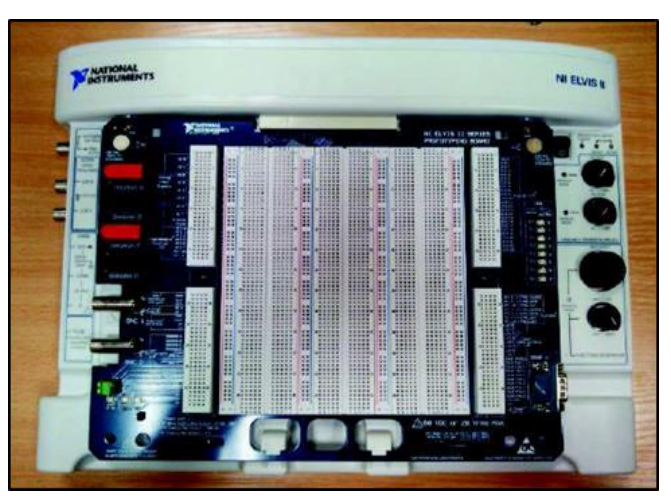

Figure 4. NI ELVISS II

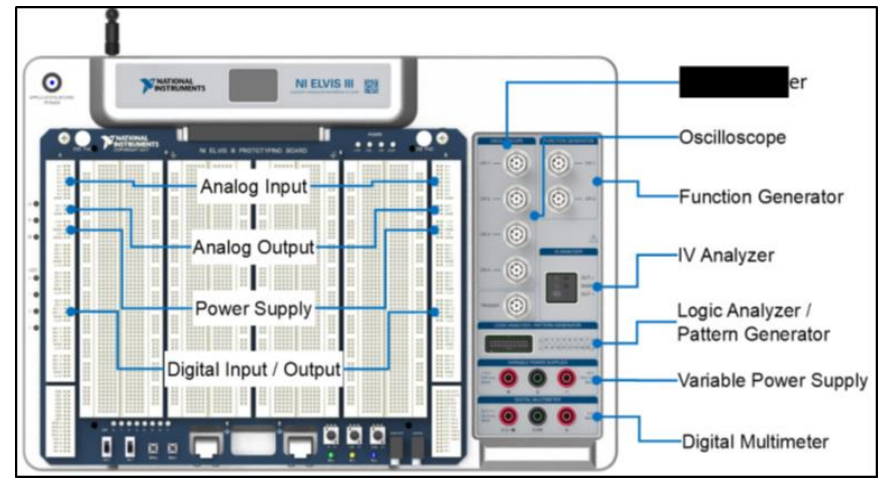

Figure 5. Integrated module of NI Eleviss

\section{EMBEDDED SYSTEM+WEB SERVICES VS LABVIEW+NI ELVIS}

\subsection{Architecture}

In architecture of S1 we used 2 web servers of reason that the unit of control and command of the practical work is an electronic card Rasppebery or a PCDUINO Card this card have limited capacity at the level of network bandwidth and the memory RAM and ROM, and since our laboratory is managed by a management platform linked to information system of university and our system know a big traffic of visitor so we need to have a real web server is where we have our management platform and information system and the second server is PCduino card is just for control and command the practical work. In the $2^{\text {nd }}$ architecture 
of S2 we used a single server which presents more services it is a true server with configurable parameter of TCP and security, the control part command and analysis it is integrated on Labview.

\subsection{Development and performance}

Graphic programming with labview facilitates and accelerates the development of the front panel of the practical work but for some task requires to have a good level (level CLAD) in the programming labview because there is several rule to respect and you must know the internal architecture of labview on the other hand programming with the javascript language and technology NODE JS it easy but takes time for the first time but after the method only repeats itself so generally the development with the languages of high levels like javascript is more flexible and powerful.

\subsection{Comparison the requirements that must have the users of S1 and S2}

For E@SLab version, S2 the client must install the NI Labview run-time engine that exists on the NI website of NI and the community of NI insists and recommend the user to use the internet explorer browser. On the other hand for S1 users are free to choose their browser and there is no obligation to install. General differences are described in the following Table 1 in Appendix.

\section{RELATED WORK VISIR AND NETLAB VS E@SLAB}

The new thing that brings our E@Slab (s1 and s2) system compared to the other famous remote laboratory system like VISIR and NetLab $[9,10]$. It was the architecture and development technology cost and flexibility:

\subsection{Real time application and architecture}

Regarding architecture: in our system as it is explained in the section above, in the control and command part we have used a single server which is directly linked to the instruments for to command, on the other hand in VIZIR and Netlab there is 2 to 3 server that intervenes and communicate them before to order the instruments and you know in the TCP/IP standards when we have more than transitions of http requests between the servers a time delay is added and the security fails increases. Then the remote laboratories must be real time applications that is to say the time delay must be negligible.

\subsection{LABVIEW and Flash vs NODE JS}

Choice the technology of development are very important in remote laboratory systems: VISIR and NETLAB these 2 system are based on LABVIEW, It is a graphical programming language that facilitates and accelerates the programming, but using labview need a license of NI, so it is not free, on the other hand E@Slab is developed by the framework NODE js which free and easy to use in programming. In our system E@Slab Student will choice electronic component of the circuit and wiring them for remote real Assembly in laboratory via the web. To have animation on the user interface for student can drag and drop devices and wiring them, we choice to use Canvas of html5 for to have compatibility with all web browser, in VISIR we have the problem of compatibility as shown in Figure 6.

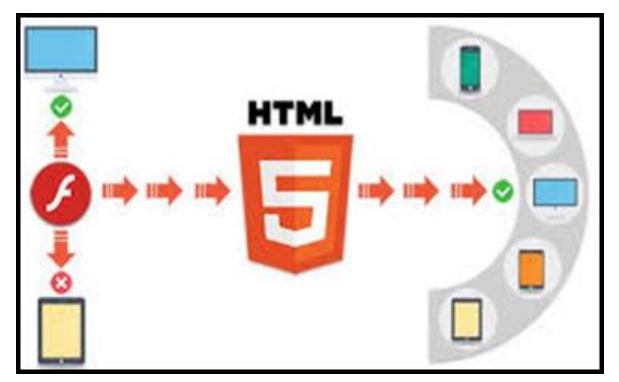

Figure 6. Html5 vs flash

\subsection{Flexibility}

Despite labview facilitates and accelerates development, there are certain tasks that require a thorough knowledge in labview at least the level of CLAD on the other hand with javascript for a problem there are several methods to solve it because it's a high level language of programation based on instructions. 


\subsection{Workspace in E@SLAB}

for controlled the practical work and create an assembly for this PW such as in VISIR and NETLAB they developed a valid application only for a single practical work of electronics but in electronic we have a lot of example of PW educational for that our system is a web application like an electronic simulator used for all practical worl as shown in Figure 3, but the difference is that it is used in the web and she controlled real electronic component that allows to do any practical work of electronics it is enough to add relays suitable for liaison members that the student must do.

\section{CONCLUSION}

An overview on process frequently used in e-learning for remote laboratory has been described in this paper. The important deciding criteria to choice between the different solutions are (i) the practical work; (ii) Skills level of developer in Web developing and technologie of LabVIEW; and (iii) the costs. The security of equipment and safety in an online experiment is also have an important role in choosing one of these technologies. After an analyst of advantages of each system and of the 2 version of E@slab, we have focused our attention on Web programing. This solution collect all advantages solutions described in this paper. Also, it stands out for its interoperability with other software thus making it suitable for the development of a variety of applications.

\section{APPENDIX}

Table 1. S1 vs S2

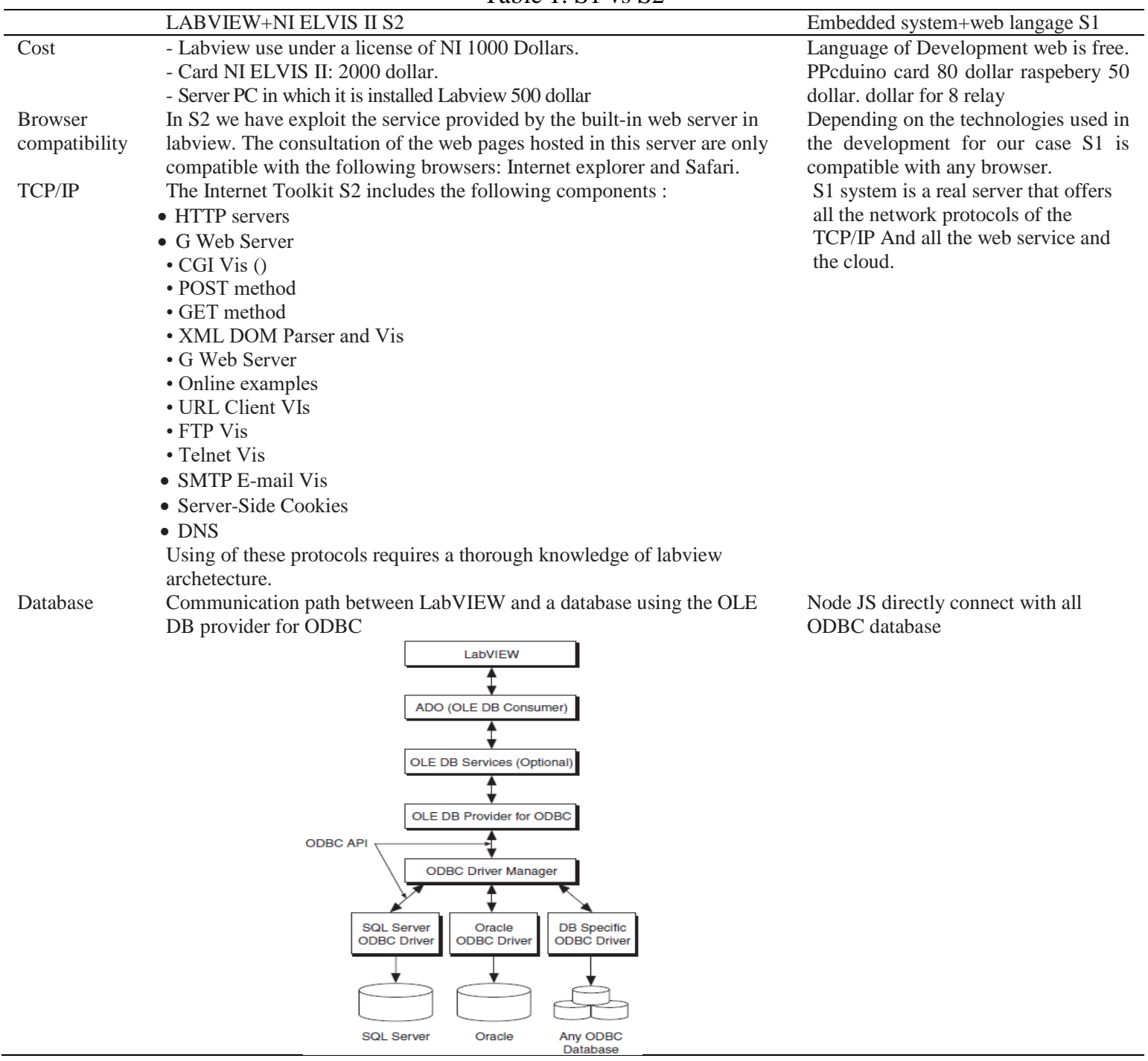

Online instrument systems in reality for remote wiring and measurement of electronic in ... (Fahd Ouatik) 


\begin{tabular}{|c|c|c|c|}
\hline & \multicolumn{2}{|c|}{ LABVIEW+NI ELVIS II S2 } & Embedded system+web langage S1 \\
\hline Security & \multicolumn{2}{|c|}{$\begin{array}{l}\text { LabVIEW does not work with standard HTTP input and output, a } \\
\text { CGI VI receives the standard input data as a string when it receives } \\
\text { a request and sends data that it generates as a string. during this } \\
\text { phase it does not present encryption algorithm } \\
\text { - The labVIEW server configuration offers limit options. }\end{array}$} & $\begin{array}{l}\text { Free to use everything of security methods, } \\
\text { protocols and encryption algorithm }\end{array}$ \\
\hline Acquisition & $\begin{array}{ll} & \text { Acquiring data } 1 \\
& \text { NI-488.2) } \\
\text { - } & \text { Deploying to NI } \\
& \text { Module) } \\
\text { - } & \text { CompactRIO pl } \\
\text { - } & \text { Multifunction I/ } \\
\text { - } & \text { PXI Multifuncti } \\
& (601 x / 602 x / 603 \\
\text { - } & \text { CompactDAQ C } \\
\text { - } & \text { C series module } \\
\text { - } & \text { CompactRIO } \\
\text { - } & \text { Digital I/O Devi } \\
\text { - } & \text { SC Express } \\
\text { - } & \text { Vehicle commu } \\
\text { - } & \text { Express/USB) } \\
\text { - } & \text { I2C/SPI interfac } \\
\text { - } & \text { USRP-RIO 29x } \\
\text { - } & \text { Ettus research B } \\
\text { - } & \text { ATCA-3671 } \\
\text { - } & \text { USB } \\
\text { - } & \text { Ethernet } \\
\text { - } & \text { Wifi }\end{array}$ & $\begin{array}{l}n \text { third-party hardware (NI-VISA, NI-Serial, } \\
\text { GA-based hardware (LabVIEW FPGA } \\
\text { Jevices (600x) } \\
\text { I/O Modules and Multifunction I/O Devices } \\
\text { 04x/605x/607x, E and B Series) } \\
\text { ssis (USB, Ethernet, Wireless) } \\
\text { II-DAQmx driver support) } \\
\text { ation buses-CAN/LIN/FlexRay (PCI/PCI } \\
\text { evice } \\
\text { TwinRX) } \\
0, \text { N210 }\end{array}$ & $\begin{array}{ll}\text { - } & \text { Digital I/O pin } \\
\text { - } & \text { Analog I/O pin } \\
\text { - } & \text { Serial comunication } \\
\text { - } & \text { I2C communication } \\
\text { - } & \text { SPI comunication } \\
\text { - } & \text { USB } \\
\text { - } & \text { Ethernet } \\
\text { - } & \text { Wifi } \\
\text { - } & \text { blutooth }\end{array}$ \\
\hline Instruments & scope & $\checkmark$ & to developed \\
\hline \multirow[t]{5}{*}{ Measurement } & Multimeter & $\checkmark$ & to developed \\
\hline & Voltmeter & $\checkmark$ & to developed \\
\hline & Ammeter & $\checkmark$ & to developed \\
\hline & Power supplier & $\checkmark$ & to developed \\
\hline & Function generator & $\checkmark$ & to developed \\
\hline Community & & $\begin{array}{l}\text { - Labview presents a great and big } \\
\text { community that shares solutions and } \\
\text { discusses issues. } \\
\text { - The NI Elvis card does not have a great } \\
\text { user community except the NI website. }\end{array}$ & $\begin{array}{l}\text { - NODE js presents a great community that } \\
\text { shares the solutions and discusses. } \\
\text { - PCDUINO and Raspebery presents a great } \\
\text { community that shares the solutions and } \\
\text { discusses. }\end{array}$ \\
\hline \multicolumn{2}{|c|}{ Speed of development } & $\begin{array}{l}\text { General LabVIEW is a graphic language } \\
\text { development is faster But some task requires } \\
\text { a good level in LabVIEW }\end{array}$ & $\begin{array}{l}\mathrm{S} 1 \text { it is developed by node js, html5, css } 3 \\
\text { JavaScript, php So the high level language } \\
\text { takes time but they have more flexible and } \\
\text { presents a freedom in the programming more } \\
\text { there are high level languages (compiled and } \\
\text { interpreted) }\end{array}$ \\
\hline \multicolumn{2}{|c|}{ Difficulty of development } & $\begin{array}{l}\text { No difficulty because several measuring } \\
\text { instrument exists by default in the ELVISS } \\
\text { card it is only necessary to control it by } \\
\text { LabVIEW. }\end{array}$ & $\begin{array}{l}\text { It is necessary to realize and to build all the } \\
\text { circuit of measurements osciloscope } \\
\text { multimétre based on the electronic carc } \\
\text { pcduino or raspebery Plus the electronic } \\
\text { components. After development of graphica } \\
\text { interface for control }\end{array}$ \\
\hline \multicolumn{2}{|c|}{ Collaborative practical work } & exists by default in LabVIEW VIs & To developed \\
\hline
\end{tabular}

\section{REFERENCES}

[1] N. Sousa, et al., "An Integrated Reusable Remote Laboratory to Complement Electronics Teaching," IEEE Transactions on Learning Technologies, vol. 3, pp. 265-271, 2010.

[2] F. Ouatik, et al., "Modeling collaborative practical work processes in an E-learning context of engineering electric education," Indonesian Journal of Electrical Engineering and Computer Science (IJEECS), vol. 16, no. 3, pp. 1464-1473, 2019.

[3] F. Ouatik, et al., "Online Engineering Education in Electronics," Journal of Materials Education, vol. 41, no. 5-6, 2019.

[4] S. Farah, et al., "Real-Time Microwav Remote Laboratory Architecture," 2015 European Microwave Conference (EuMC), Paris, 2015, pp. 1315-1318.

[5] M. Gericota, et al., "The EOLES project Remote labs across the Mediterranean," 2014 11th International Conference on Remote Engineering and Virtual Instrumentation (REV), Porto, 2014, pp. 211-216.

[6] F. Ouatik, et al., "The EOLES project remote labs across the Mediterranean: an example of a successful experience," Proceedings of the 2017 International Conference on Smart Digital Environment, 2017, pp. 155-161.

[7] F. Schauer, et al., "Easy to build Remote laboratory with data transfer using ISES-Internet School Experimental System," European Journal of Physics, vol. 29, pp. 1-13, 2008. 
[8] O. Okoyeigbo, et al., "Design and implementation of a java based virtual laboratory for data communication simulation," International Journal of Electrical and Computer Engineering (IJECE), vol. 10, no. 6, pp. 5883-5890, 2020.

[9] J. Cuadros, et al., "Educational Data Mining in an Open-Ended Remote Laboratory on Electric Circuits, Goals and Preliminary Results," Proceedings of the 8th International Conference on Educational Data Mining, 2015, pp. 578-579.

[10] M. Albu, et al., "Embedding Remote Experimentation in Power Engineering Education," IEEE Transactions on Power Systems, vol. 19, no. 1, pp. 139-143, 2004.

[11] M. Tawfik, et al., "Virtual Instrument Systems in Reality (VISIR) for Remote Wiring and Measurement of Electronic Circuits on Breadboard," IEEE Transactions on Learning Technologies, vol. 6, no. 1, pp. 60-72, 2013.

[12] M. Phanupong, et al., "Prediction of Student droput using personel profile and data mining aproch," Intelligent and Evolutionary Systems, vol. 5, pp. 143-155, 2015.

[13] R. Conjin, et al., "Predicting Student Performance from LMS Data: A Comparison of 17 Blended Courses Using Moodle LMS," IEEE Transactions on Learning Technology, vol. 10, no. 1, pp. 17-29, 2017.

[14] J. Garcia-Zubia, "Empirical Analysis of the Use of the VISIR Remote Lab in Teaching Analog Electronics," in IEEE Transactions on Education, vol. 60, no. 2, pp. 149-156, 2017.

[15] R. Hubscher, "Assigning Students To Groups Using General And Context-Specific Criteria," in IEEE Transactions On Learning Technology, vol. 3, no. 3, pp. 178-189, 2010.

[16] N. Ben Amor, et al., "Naive Bayes vs Decision Trees in Intrusion Detection Systems," in Proceedings ACM Symposium on Applied Computing, 2004, pp. 420-424.

[17] C. Lakshmi Devasena, et al., "Effectiveness Evaluation of Rule Based Classifiers for the Classification of Iris Data Set," Bonfring International Journal of Man Machine Interface Special, vol. 1, pp. 5-9, 2011.

[18] J. Lu, et al., "Recommender system application developments: A survey," Decision Support Systems, vol. 74, pp. 12-32, 2015.

[19] Merdt, et al., "Evaluating Recommender Systems for Technology Enhanced Learning: A Quantitative Survey," IEEE Transactions on Learning Technologies, vol. 8, no. 4, pp. 326-344, 2015.

[20] Fouatik, et al., "Comparative study of MapReduce classification algorithms for students orientation," Procedia Computer Science, vol. 170, pp. 1192-1197, 2020.

[21] S. Farah, et al., "Multi-User and Real-Time Flexible Remote La-boratory Architecturefor Collaborative and Cooperative Pedagogical Scenarios," International Journal of Online and Biomedical Engineering (iJOE), vol. 12, no. 4, pp. 33-36, 2016.

[22] J. García-Zubia, "Towards a Distributed Architecture for Remote Laboratories," International Journal of Online Engineering (iJOE), vol. 4, no. 1, pp. 11-14, 2008.

[23] N. Hida, et al., "Components of Participatory Engagement within E-Learning Community," Indonesian Journal of Electrical Engineering and Computer Science (IJEECS), vol. 12, no. 2, pp. 556-561, 2018.

[24] A. Mahmoud, et al., "The patterns of accessing learning management system among students," Indonesian Journal of Electrical Engineering and Computer Science (IJEECS), vol. 13, no. 1, pp. 15-21, 2019.

[25] A. Akmal, “Assessing Students' Continuance Intention in Using Multimedia Online Learning," TELKOMNIKA Telecommunication, Computing, Electronics and Control, vol. 17, no. 1, pp. 187-193, 2019.

[26] M. Gasah, et al., "An approach in creating positive emotion for children's e-learning based on user interface design," Indonesian Journal of Electrical Engineering and Computer Science (IJEECS), vol. 13, no. 3, pp. 1267-1273, 2019.

[27] S. Sfenrianto, et al., "E-Learning Effectiveness Analysis in Developing Countries: East Nusa Tenggara, Indonesia Perspective," Bulletin of Electrical Engineering and Informatics (BEEI), vol. 7, no. 3, pp. 417-424, 2018.

[28] S. K. Bhuyan, et al., "Modeling and Simulation of Grid Connected Hybrid Energy System and its Fault Analysis," International Journal of Power Electronics and Drive Systems (IJPEDS), vol. 9, no. 2, pp. 775-783, 2018.

[29] E. Akanksha, "Framework for propagating stress control message using heartbeat based IoT remote monitoring analytics," International Journal of Electrical and Computer Engineering (IJECE), vol. 10, no. 5, pp. 4615-4622, 2020.

[30] I. Matazi, et al., "Development of Intelligent Multi-agents System for Collaborative e-learning Support," Bulletin of Electrical Engineering and Informatics (BEEI), vol. 7, no. 2, pp. 294-305, 2018. 